\title{
Mineração
}

\section{Tecnologia limpa para redução de impacto ambiental do cianeto na mineração de ouro}

\author{
Josiani Costa Riani \\ Ex-bolsista do Núcleo de Valorização em Materiais Minerais da UFOP, \\ Doutoranda do Programa em Engenharia de Metalúrgica e de Materiais - USP \\ E-mail:josiani.riani@poli.usp.br \\ Pablo dos Santos Pina \\ Doutorando do Programa de Pós-Graduação da REDEMAT \\ E-mail: pablopinabr@yahoo.com.br \\ Versiane Albis Leão \\ Professor do Departamento de Engenharia Metalúrgica e de Materiais/UFOP \\ E-mail:versiane@demet.em.ufop.br
}

\section{Resumo}

Esse trabalho discute a aplicação de resinas de troca iônica com matriz poliacrílica para a adsorção de cianocomplexos metálicos. Ensaios em batelada mostraram que as resinas possuem elevada afinidade para os cianocomplexos de ferro e de zinco em relação aos de cobre. Já a eluição, estudada em coluna, indicou que soluções de tiocianato de sódio a 1mol/L são capazes de eluir os ciancomplexos dos três metais. Já soluções de nitrato de sódio, na mesma concentração, não são efetivas para a eluição do cianeto de zinco. Um processo de eluição seletiva é proposto onde os cianocomplexos de cobre e de ferro são eluídos com solução íons $\mathrm{NO}_{3}^{-}$. Em seguida, o zinco é eluído com NaSCN. Ambos eluentes foram capazes de concentrar os cianocomplexos de solução, o que tem efeito benéfico nas etapas subseqüentes de regeneração do cianeto.

Palavras-chave: Cianetos metálicos, reciclagem, resinas de troca iônica, resinas poliacrílicas.

\begin{abstract}
A study on metal cyanocomplexes sorption on polyacrylic ion exchange resins has been undertaken. Batch sorption experiments have shown that polyacrylic resins have a high affinity for zinc and iron cyanides compared to copper complexes. Elution studies performed in column experiments have also depicted that $1 \mathrm{mo} / \mathrm{L}$ sodium thiocyanate solutions can successfully extract the three metals. Conversely, sodium nitrate solutions at the same concentration are not effective in eluting zinc cyanocomplexes. Based on elution figures, it is proposed that copper and iron can be selectively eluted with nitrate before the zinc, this latter being recovered afterwards with thiocyanate solutions. Both eluants were able to concentrate the cyanocomplexes in solution with a beneficial effect on the subsequent steps of cyanide recycling
\end{abstract}

Keywords: Cyanide, recycling, ion exchange resins, polyacrylic resins. 


\section{Introdução}

Os compostos de cianeto são utilizados em diferentes setores da indústria, sendo que $80 \%$ da produção destina-se à manufatura de plásticos tais como o náilon, tecidos para a área médica e fármacos. No setor de materiais, o cianeto está presente em processos de recobrimento de superfícies, no tratamento térmico de aços, em aplicações fotográficas e produção de borracha sintética. Os outros $20 \%$ são utilizados, na forma de cianeto de sódio, principalmente na a mineração de ouro ao redor do mundo (Logsdon et al., 1999).

O uso do cianeto de sódio na mineração de ouro vem da sua habilidade em reagir com o metal, além da prata, formando complexos estáveis em soluções aquosas. Entretanto, devido à toxicidade intrínseca do agente lixiviante, os processos de cianetação vem sendo questionados do ponto de vista de seu risco ambiental (Sengupta, 1992).

Ambientalmente, efluentes contendo cianeto apresentam riscos para: (i) mamíferos (ii) répteis (iii) anfíbios (iv) aves (especialmente as migratórias) (v) peixes e vida aquática em geral. Uma vez que os efluentes são tratados em áreas restritas, cercas e outros tipos de barreiras impedem o acesso de animais (mamíferos, répteis e anfíbios) aos efluentes. A principal preocupação é com a vida aquática (peixes e outros). Embora espécies sensíveis, como trutas, apresentem certa tolerância às formas toxiologicamente ativas do cianeto, a vida aquática é a primeira e uma das mais atingidas quando exposta a efluentes que o contêm. Esse fenômeno é corroborado pelos acidentes ocorridos nas minas de Omai (Guiana), em agosto de 1995, e de Aural (Romênia), em janeiro de 2000, que tiveram grande impacto sobre a vida aquática. No caso da mina de Aural, foram lançadas cerca de 100 toneladas de cianeto em um afluente do rio Danúbio, com grande cobertura da impressa mundial. Tal acidente causou a morte de toneladas de peixes e dois milhões de pessoas tiveram contato com água contaminada (Miller e Pritsos, 2001, DeVries, 2001).
No caso brasileiro, a redução do impacto ambiental do cianeto pela mineração do ouro envolve a degradação natural, onde o cianeto decompõe-se para o íon cianato ( $\left.\mathrm{CNO}^{-}\right)$. Nessa forma de tratamento, a participação de microrganismos é responsável pela catálise da reação de degradação. O cianeto também pode ser degradado por processos químicos oxidativos (oxidação por hipoclorito, por ozônio, por misturas $\mathrm{SO}_{2}$ /ar e por peróxido de hidrogênio). Outra prática é a sua complexação com íons ferro formando compostos da família do azul da Prússia que o removem por precipitação. Os íons ferrocianeto apresentam elevada estabilidade e seus precipitados, baixa solubilidade. Entretanto a possível fotodegradação (com liberação de íons cianeto) desses precipitados vem sendo discutida na literatura científica (Young, 2001).

Em função dos dois acidentes previamente discutidos, o "International Cyanide Management Code” impôs, recentemente, um limite de liberação de cianeto WAD ("weak acid dissociable", cianocomplexos de zinco, níquel e 2/3 do cianocomplexo de cobre) de até 50mg/L nas soluções enviadas para as barragens de rejeitos. Essa diretiva reforça a necessidade de se utilizarem métodos para redução do impacto ambiental do cianeto já dentro das próprias usinas produtoras. Além das alternativas envolvendo destruição, uma outra opção é a reciclagem. Nessa última, alia-se a vantagem da redução da concentração de cianeto nos efluentes com a redução dos custos de compra do agente lixiviante (o cianeto é o reagente de maior custo no processo de cianetação). Atualmente, a reciclagem de cianeto tem sido limitada ao processamento de minérios que apresentam elevado consumo de cianeto (minérios contendo prata e cobre) (Ciminelli, 2002). Entretanto pode-se vislumbrar uma crescente aplicação desse princípio a minérios de outras tipologias.

Resinas de troca iônica são uma opção para a reciclagem de cianeto, pois apresentam as seguintes vantagens: (i) carregam facilmente cianetos metálicos; (ii) podem ser aplicadas tanto em soluções clarificadas como em polpas; (iii) os cianocomplexos metálicos são concentrados várias vezes, o que permite a obtenção de um eluato com elevada concentração de cianeto. Isto reduz custos e melhora as condições de segurança nas etapas subseqüentes do processo de reciclagem (Fleming, 2001). Leão et al. (2000) estudaram a adsorção de cianocomplexos metálicos em resinas de troca iônica, em soluções com altos níveis de cobre. Foram utilizados sistemas com concentração fixa dos metais cobre, ferro e níquel. Observou-se um elevado carregamento de cobre e ferro em resinas de matriz poliacrílica. Entretanto, o efeito de diferentes concentrações dos metais sobre a eficiência de adsorção não foi avaliado. Os estudos de eluição foram conduzidos sempre numa mesma concentração de eluante (Lukey et al., 2000; Leão \& Ciminelli, 2001; Lukey et al., 2001). Outro parâmetro ainda pouco avaliado é o efeito da temperatura sobre a eluição dos metais.

O objetivo desse trabalho foi estudar adsorção na resina Bayer AP247 (macroporosa, matriz poliacrílica, trimetilamônio quaternário como grupo de troca) em soluções com diferentes concentrações de cianocomplexos, que simulam efluentes da lixiviação de ouro. Avaliouse, também, a sua eluição com dois tipos de eluentes ( $\mathrm{NaSCN}$ e $\mathrm{NaNO}_{3}$ ). Os resultados dos experimentos de adsorção mostram que o carregamento da resina depende da concentração dos complexos em solução, mas foi observada uma alta afinidade para o cianocomplexo de ferro. O reagente NaSCN pode ser usado para eluir resinas com matriz poliacrílica, alcançando recuperações metálicas acima de $90 \%$, a $50^{\circ} \mathrm{C}$. Já o $\mathrm{NaNO}_{3}$ elui com eficiência os cianocomplexos de cobre e de ferro da resina estudada, atingindo recuperações acima de 90\%. Entretanto a eluição do zinco é muito lenta, ou seja, atinge uma recuperação de $28 \%$ $\left(50^{\circ} \mathrm{C}\right)$ apenas depois de passados 20 volumes de leito através da coluna. 
Josiani Costa Riani et al.

\section{Metodologia Adsorção}

Os experimentos foram realizados utilizando-se soluções sintéticas produzidas a partir da solubilização de cianeto de cobre (CuCN, 99\%, Aldrich), ferrocianeto de potássio triidratado $\left(\mathrm{K}_{4} \mathrm{Fe}(\mathrm{CN})_{6} \cdot 3 \mathrm{H}_{2} \mathrm{O}, 98,5 \%\right.$, Isofar $)$ e sulfato de zinco heptidratado $\left(\mathrm{ZnSO}_{4} \cdot 7 \mathrm{H}_{2} \mathrm{O}\right.$, 99\%, Synth) em cianeto de sódio ( $\mathrm{NaCN}$, 95\%, Synth) para os cianocomplexos metálicos. As concentrações dos metais nas soluções variaram entre 50 e $500 \mathrm{mg} / \mathrm{L}$, em pH 10,5 $\pm 0,5$. Dessa maneira, pôde-se avaliar o comportamento da adsorção dos cianocomplexos para diferentes concentrações em solução.

Primeiramente, a resina foi ativada em solução 1mol/L de KCl por um período de 24 horas. Os experimentos foram realizados à temperatura de $25^{\circ} \mathrm{C}$, em erlenmeyers. A adsorção, em função do tempo, foi avaliada através da retirada de amostras a 10, 30, 60, 120 e 480 minutos, da seguinte forma: para cada tempo de adsorção, $1 \mathrm{~mL}$ de resina era contactado com $100 \mathrm{~mL}$ de solução. O pH da solução era ajustado com $\mathrm{NaOH}$ para $10,5 \pm 0,5$. Ao final de cada tempo de adsorção, o erlenmeyer era retirado da mesa oscilatória, sendo que a mistura resinasolução era filtrada e a fase aquosa, analisada quanto a seu teor de cobre, de ferro e de zinco. O carregamento da resina $\left(\mathrm{q}_{\mathrm{eq}}\right)$ foi determinado através de balanço de massa para a solução antes e após o experimento (eq. 1):

$\mathrm{q}_{\text {eq }}=\frac{\left(\mathrm{C}_{0}-\mathrm{C}_{\mathrm{t}}\right) \cdot \mathrm{V}_{\text {solução }}}{\mathrm{V}_{\text {resina }}}$

Onde:

$\mathrm{C}_{0}$ é a concentração inicial do metal em solução, $C_{t}$ é a concentração do metal no instante t, V é o volume de solução $(0,1 \mathrm{~L})$ e $\mathrm{V}_{\text {resina }}$ é o volume de resina $(1 \mathrm{~mL})$.

\section{Eluição}

Anteriormente aos experimentos de eluição, a resina foi contatada com uma solução sintética ( $\mathrm{pH} 10,5 \pm 0,5)$ com a seguinte concentração, 300mgFe/L, 200mgCu/L e 180mgZn/L, em mesa oscilatória. Essa solução foi preparada de maneira semelhante à utilizada nos ensaios de adsorção. Ao final de 24 horas, a mistura resina-solução foi separada por filtração, sendo a fase aquosa analisada quanto a seus teores de cobre, de ferro e de zinco. O carregamento médio da resina foi obtido pela diferença da concentração inicial e final da solução, segundo a Equação 1, sendo obitidos as seguintes valores: 11,0mgFe/mL-resina, 8,8mgCu/mL-resina e 7,3mgZn/mL-resina.

Os experimentos de eluição foram conduzidos em uma microcoluna, nas temperaturas de $25^{\circ} \mathrm{C}$ e $50^{\circ} \mathrm{C}$, sendo a coluna aquecida por recirculação de água, utilizando-se uma bomba peristáltica e um banho termostatizado. A coluna era alimentada com um leito de $10 \mathrm{~mL}$ de resina e a solução de eluição foi passada em fluxo ascendente de 0,08mL/min/mL-resina, também por uma bomba peristática. Foram passados, através da coluna, um total de 20 volumes de leito (BV). As soluções de eluição foram preparadas com concentração de $1 \mathrm{~mol} / \mathrm{L}$ de $\mathrm{NaNO}_{3}$ (99\%, Synth) ou NaSCN (99,9\%, Merck) em água destilada contendo entre 300 $\mathrm{mgCN}^{-} / \mathrm{L}$ e 400 $\mathrm{mgCN}^{-} / \mathrm{L}$, em pH 11,0-11,5.

Em todos os experimentos, a concentração dos metais em solução foi determinada através da técnica de espectroscopia de absorção atômica, usando o equipamento Perkin-Elmer, modelo AAnalyst 100.

\section{Resultados e discussão Adsorção}

Os carregamentos dos cianocomplexos de cobre, de zinco e de ferro na resina estão apresentados nas Figuras 1(a) a 1(c). Esses gráficos apresentam o carregamento relativo (razão da concentração de metal na resina, pela concentração de metal inicialmente em solução) como uma função do tempo. A ordem de carregamento para a resina Bayer AP247 foi diferente a cada concentração dos metais em solução.

Analisando os carregamentos, constatou-se que, em menores concentrações do cianocomplexo de ferro (50mg/L), Figura 1(a), havia um maior carregamento relativo do metal e, em todas as outras concentrações (Figuras 1(b) e 1(c)), o carregamento relativo do cianocomplexo de zinco foi maior do que dos outros cianocomplexos presentes.

Para a concentração, inicial em solução, de 500mgZn/L, 100mgCu/L e $50 \mathrm{mgFe} / \mathrm{L}$ (Figura 1(a)), nota-se que todo o ferro foi carregado e cerca de $67 \%$ e $65 \%$ de zinco e de cobre se adsorveram, respectivamente. Riveros (1993) realizou experimentos de adsorção em uma solução contendo $5 \mathrm{mgAu} / \mathrm{L}, 18 \mathrm{mgCu} / \mathrm{L}$, $9 \mathrm{mgFe} / \mathrm{L}, 14 \mathrm{mgNi} / \mathrm{L}$ e $8 \mathrm{mgZn} / \mathrm{L}$, em resina Amberlite IRA-458 (matriz de poliacrilamida) semelhante à matriz da resina Bayer AP247. Ao final do experimento o autor obteve o seguinte carregamento: 10mgZn/mL-resina, 10,2mgFe/mL-resina e 8,6mgCu/mL-resina. Foi observado um carregamento de ferro e de zinco maior do que o carregamento de cobre, apesar de a concentração de cobre em solução ser maior que a concentração dos dois metais, confirmando a maior afinidade para o zinco e ferro em relação ao cobre. Da mesma forma,Vachon (1985) observou uma menor afinidade da resina Amberlite IRA 958 (matriz poliacrília) para o cobre em relação ao ferro e ao zinco em experimentos em coluna. Segundo o autor, as diferentes afinidades da resina pelos metais puderam ser observadas através do perfil de carregamento da resina. No caso do cobre, o "overshooting" do metal foi observado (sua concentração no efluente tornou-se maior do que na solução que entrava na coluna), o que foi associado à sua substituição pelo ferro. O mesmo não pôde ser observado em relação ao zinco. Os carregamentos obtidos foram: 1,7-2,5mgFe/ mL-resina, 12,5-13,0mgCu/mL-resina e 02,6mgZn/mL-resina a partir de um efluente contendo em média: $28 \mathrm{mgFe} / \mathrm{L}$, 64mgZn/L e 190mgCu/L. 
Analisando a ordem de carregamento relativo da solução contendo 500mgFe/L, 100mgZn/L e 50mgCu/L (Figura 1(b)), é observado que cerca de $70 \%$ de zinco é carregado, seguido por $39 \%$ de Cu e 35\% de ferro, isto é, nessas condições, observa-se um carregamento relativo de cobre e ferro muito próximos e menores do que o de zinco. O carregamento da resina para uma concentração em solução de $500 \mathrm{mgCu} / \mathrm{L}$, 100mgFe/L e 50mgZn/L (Figura 1(c)) indicou a seguinte ordem de carregamento: $\mathrm{Zn}(75 \%)>\mathrm{Fe}(60 \%)>\mathrm{Cu}(43 \%)$. Ensaios de adsorção foram também realizados por Leão et al. (2000), utilizando a resina Purolite A860S (matriz poliacrílica, grupo de troca trimetilamônio quaternário). Para uma concentração em solução de $500 \mathrm{mgCu} / \mathrm{L}, 50 \mathrm{mgNi} / \mathrm{L}$ e $15 \mathrm{mgFe} / \mathrm{L}$, foi obtida uma ordem de carregamento de $\mathrm{Cu}(67 \%)>\mathrm{Ni}(56 \%)>\mathrm{Fe}(50 \%)$. Esse resultado confirma, assim, uma maior afinidade de resinas com matriz poliacrílica pelos cianocomplexos de zinco e de ferro em relação ao de cobre. Baseando-se nos resultados obtidos por Leão et al. (2000), seria provável, no presente trabalho, um maior carregamento do cianocomplexo de cobre em resinas com matriz poliacrílica, o que não foi observado quando o níquel foi substituído pelo cianocomplexo de zinco na solução de carregamento. Obteve-se, portanto, um maior carregamento relativo do cianocomplexo de zinco (em comparação ao cobre e ferro) para a concentração dada anteriormente. A distribuição espacial dos grupos trimetilamônio favorece o carregamento desse cianocomplexo, o que gera carregamentos elevados do metal nessa resina (Riani et al., 2002).

Comparadas às resinas de matriz de poliestireno (Riani et al., 2002), resinas de matriz poliacrílica apresentam uma maior afinidade para os cianocomplexos de ferro. Essa maior afinidade foi também observada por Riveros (1993) e Leão et al. (2001). Esse comportamento é explicado pelo caráter hidrofílico da resina Bayer AP247, a qual apresenta uma maior afinidade para os cianocomplexos mais hidratados, como o $\left[\mathrm{Fe}(\mathrm{CN})_{6}\right]^{-4}$, em relação às resinas de matriz de poliestireno. (a)

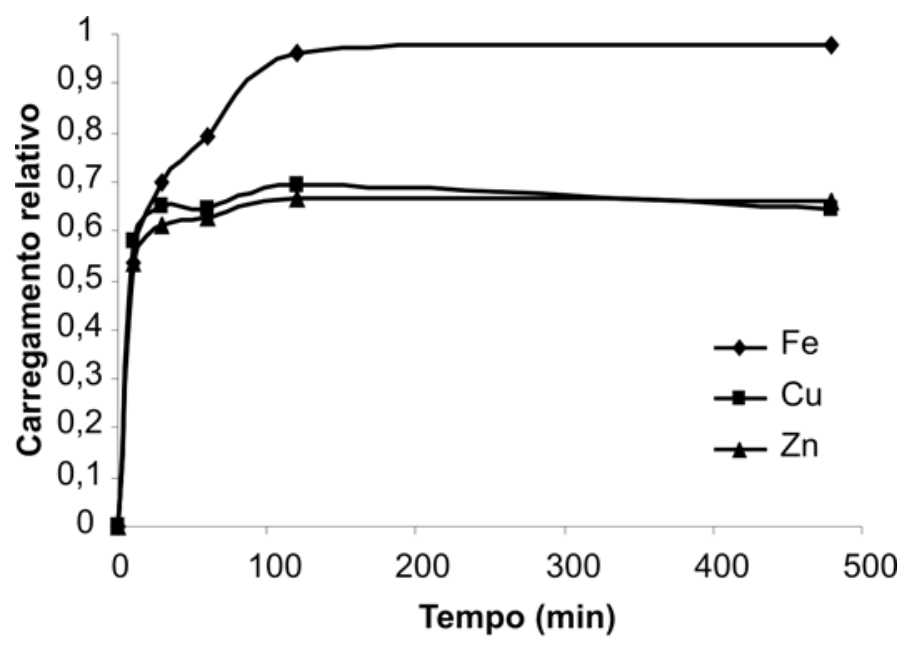

(b)

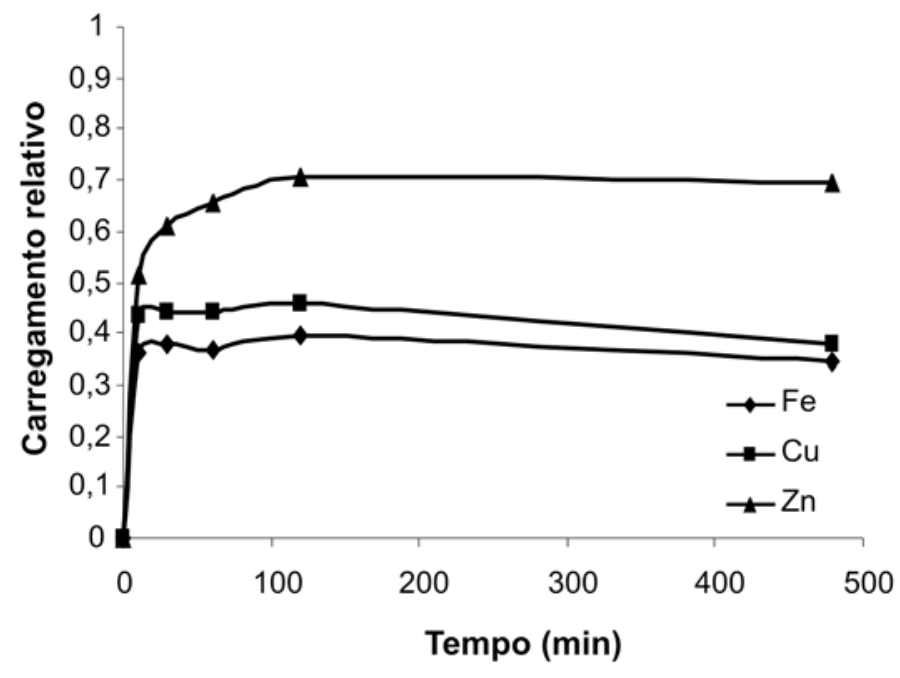

(c)

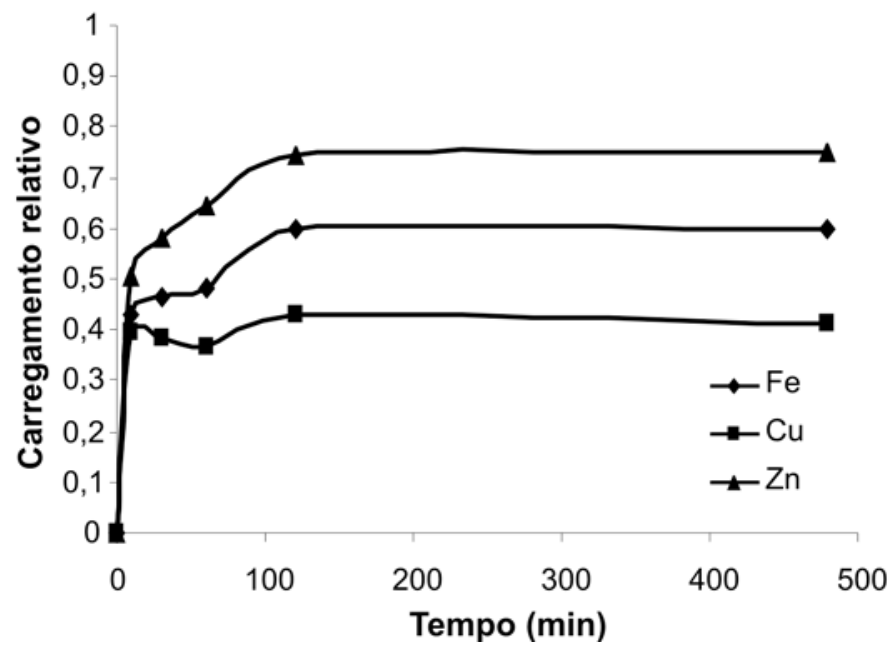

Figura 1 - Carregamento relativo dos cianocomplexos de cobre, de zinco e de ferro na resina base forte Bayer AP247 $\mathrm{pH} 10,5 \pm 0,5,25^{\circ} \mathrm{C}$. Concentração inicial da solução: $500 \mathrm{mgZn} / \mathrm{L}, 100 \mathrm{mgCu} / \mathrm{L}$ e $50 \mathrm{mgFe} / \mathrm{L}$, (a); $500 \mathrm{mgFe} / \mathrm{L}, 100 \mathrm{mgZn} / \mathrm{L}$ e $50 \mathrm{mgCu} / \mathrm{L}$, (b); $\% 00 \mathrm{mgCu} / \mathrm{L}, 100 \mathrm{mgFe} / \mathrm{L}$ e $50 \mathrm{mgZn} / \mathrm{L}$ (c). 
Josiani Costa Riani et al.

A Tabela 1 apresenta o carregamento metálico total (meq/mL-resina) da resina Bayer AP247. Observa-se que o carregamento metálico é maior para aquele metal que apresenta maior concentração em solução. De acordo com os resultados obtidos, a resina Bayer AP247, com matriz poliacrílica, é apropriada para recuperar, principalmente, os cianocomplexos de ferro e de zinco de efluentes, tendo uma recuperação que chegou a $100 \%$ do metal em algumas concentrações.

\section{Eluição}

A eluição da resina Bayer AP247 utilizando como eluente $1 \mathrm{~mol} / \mathrm{L}$ de $\mathrm{NaSCN}$, à temperatura de $25^{\circ} \mathrm{C}$, está apresentada na Tabela 2. Os resultados mostram que, nos primeiros 5 volumes de leito, cerca de $90 \%$ de cobre é eluído, não havendo crescimento na recuperação para maiores volumes. A eluição do zinco é mais lenta e atinge um valor em torno de $80 \%$ de recuperação, ao final do ensaio. Nos primeiros 5 volumes de leito, sua recuperação foi de 53\%. A eluição do ferro atinge $95 \%$ nos primeiros 5 volumes de leito, mantendo-se constante nesse valor até o final do ensaio. Aumentando a temperatura para $50^{\circ} \mathrm{C}$, notase que o cianocomplexo de cobre atinge cerca de 92\% de recuperação, nos primeiros 5 volumes de leito, permanecendo nesse valor até o final. Comparandose com os experimentos a $25^{\circ} \mathrm{C}$, observa-se que não houve uma mudança significativa na eluição do metal. O zinco atinge $94 \%$ de recuperação, ao final de 20 volumes de leito, comparado com $80 \%$, que foi atingido à temperatura de $25^{\circ} \mathrm{C}$. O cianocomplexo de ferro não apresenta mudança na sua recuperação, quando a temperatura foi aumentada de $25^{\circ} \mathrm{C}$ para $50^{\circ} \mathrm{C}$, nos experimentos a $1 \mathrm{~mol} / \mathrm{L} \mathrm{NaSCN}$.

Os resultados dos experimentos de eluição da resina Bayer AP247 com 1mol/L $\mathrm{NaNO}_{3}$, a $25^{\circ} \mathrm{C}$, estão também apresentados na Tabela 2. Nota-se que o cianocomplexo de ferro atinge uma recuperação de $81 \%$ nos primeiros 5 volumes de leito, sendo que, ao final do ensaio, sua eluição foi cerca de $97 \%$. Por outro lado, o cianocomplexo de zinco atinge, ao fi-

Tabela 1 - Carregamento metálico total (meq/mL de resina) na resina Bayer AP247.

\section{Concentração inicial em solução (mg/L)}

Carregamento metálico total na resina (meq/mL de resina)

\begin{tabular}{c|c|c|c|c|c}
\hline $\mathrm{Fe}$ & $\mathrm{Cu}$ & $\mathrm{Zn}$ & $\mathrm{Fe}$ & $\mathrm{Cu}$ & $\mathrm{Zn}$ \\
\hline 100 & 500 & 50 & 0,4 & 0,8 & 0,1 \\
\hline 500 & 50 & 100 & 1,2 & 0,1 & 0,2 \\
\hline 50 & 100 & 500 & 0,3 & 0,3 & 1,0 \\
\hline
\end{tabular}

Tabela 2 - Eluição dos cianocomplexos metálicos da resina Bayer AP247, usando $1 \mathrm{~mol} / \mathrm{L} \mathrm{NaSCN}$ e $1 \mathrm{~mol} / \mathrm{L} \mathrm{NaNO}_{3}$ como eluentes e a temperaturas de $25^{\circ} \mathrm{C}$ e $50^{\circ} \mathrm{C}$

\begin{tabular}{|c|c|c|c|c|c|c|c|}
\hline \multirow{3}{*}{ Eluente } & \multirow{3}{*}{$\begin{array}{c}\mathbf{n}^{\circ} \mathrm{de} \\
\text { volumes de } \\
\text { leito }(\mathrm{BV})\end{array}$} & \multicolumn{6}{|c|}{ \% Eluição } \\
\hline & & \multicolumn{3}{|c|}{$25^{\circ} \mathrm{C}$} & \multicolumn{3}{|c|}{$50^{\circ} \mathrm{C}$} \\
\hline & & $\mathrm{Fe}$ & $\mathrm{Cu}$ & $\mathrm{Zn}$ & $\mathrm{Fe}$ & $\mathrm{Cu}$ & Zn \\
\hline \multirow{2}{*}{ NaSCN } & 5 & 95 & 90 & 53 & 93 & 92 & 66 \\
\hline & 20 & 95 & 90 & 80 & 96 & 92 & 94 \\
\hline \multirow{2}{*}{$\mathrm{NaNO}_{3}$} & 5 & 81 & 41 & 6 & 91 & 36 & 7 \\
\hline & 20 & 97 & 81 & 21 & 99 & 85 & 28 \\
\hline
\end{tabular}

nal de 20 volumes de leito, uma recuperação de apenas $21 \%$. Analisando a eluição do cobre, nota-se que esta alcançou, ao final do experimento, uma recuperação próxima de $81 \%$, sendo que, nos primeiros 5 volumes de leito, obteve-se um valor de $41 \%$ de eluição. Aumentando a temperatura para $50^{\circ} \mathrm{C}$, tem-se que o cianocomplexo de cobre atinge, ao final de 20 volumes de leito, uma recuperação próxima de $85 \%$. A recuperação do cianocomplexo de zinco mostrou um pequeno aumento passando de $21 \%$, à temperatura de $25^{\circ} \mathrm{C}$, para $28 \%$, à temperatura de $50^{\circ} \mathrm{C}$, depois de passados 20 volumes de leito através da coluna. Analisando a eluição do ferro, nota-se que esta não foi significativamente afetada pelo aumento da temperatura, nessa concentração de $\mathrm{NaNO}_{3}$, ou seja, passou de $97 \%$ $\left(25^{\circ} \mathrm{C}\right)$ para $99 \%\left(50^{\circ} \mathrm{C}\right)$.

Ainda segundo a Tabela 2, observa-se que a eluição final do cianocomplexo de ferro não é afetada, nem pela mudança do reagente, nem pelo aumento da temperatura. Sua recuperação é sempre superior a 95\%. A recuperação de cobre sofre uma ligeira redução, quando eluído com soluções de íons nitrato em comparação com tiocianato. Por outro lado, a recuperação do zinco é, significativamente, elevada ao se substituir $\mathrm{NaNO}_{3}$ por NaSCN. Porém o efeito da temperatura na eluição do zinco é menos expressivo do que a substituição do eluente.

Vachon (1985) realizou experimentos de eluição na resina Amberlite IRA958 (matriz poliacrílica), semelhante à resina Bayer AP247, utilizando soluções a $15 \% \mathrm{NaCl}$ como eluente. Os resultados dos experimentos mostraram que cerca de $97 \%$ de ferro e $83 \%$ de cobre foram eluídos. Leão e Ciminelli (2000) realizaram experimentos de eluição com $2 \mathrm{~mol} / \mathrm{L}$ $\mathrm{NaCl}$, na resina Purolite A860S (matriz poliacrílica), e notaram que cerca de $71 \%$ de ferro e $84 \%$ de cobre foram eluídos. Isto sugere que a eluição com $\mathrm{NaNO}_{3}$ (menor concentração) é mais efetiva do que com $\mathrm{NaCl}$ e menos eficaz do que com NaSCN, para resinas poliacrílicas. 
O objetivo do uso de resinas de troca iônica é a concentração dos cianocomplexos no eluato e com isso facilitar a etapa seguinte de reciclagem do cianeto. Conseqüentemente, a concentração dos complexos no eluente é um parâmetro importante na regeneração. Os perfis de eluição da resina Bayer AP247, usando como eluente soluções $1 \mathrm{~mol} / \mathrm{L}$ de NaSCN, à temperatura de $25^{\circ} \mathrm{C}$ e de $50^{\circ} \mathrm{C}$, estão apresentado nas Figuras 2a e 2b, respectivamente.

A eluição do ferro, na resina Bayer AP247, é mais rápida, se comparada com a dos outros metais nas duas temperaturas estudadas. É importante destacar que, devido à natureza da matriz, a eluição do ferro se dá nos primeiros 5 volumes de leito, o que gera uma concentração do metal no eluato bem superior às observadas com a resina de matriz de poliestireno (Riani et al., 2004). A concentração do metal é da ordem de 8g/L na solução eluente que deixa a coluna. A eluição do cobre é um pouco mais lenta, refletindo um perfil de concentrações no eluanto com menor concentração e, havendo, assim, a necessidade de um maior número de volumes de leito para eluição do metal. Comportamento semelhante, mas com maior magnitude, é observado para o zinco.
Os perfis de eluição da resina Bayer AP247, usando como eluente soluções $1 \mathrm{~mol} / \mathrm{L}$ de $\mathrm{NaNO}_{3}$, à temperatura de $25^{\circ} \mathrm{C}$ e $50^{\circ} \mathrm{C}$, estão apresentados nas Figuras 3(a) e 3(b).

Para os ensaios conduzidos com soluções $1 \mathrm{~mol} / \mathrm{L}$ de nitrato de sódio, observa-se uma menor concentração dos três metais no eluato. Por exemplo, a concentração de ferro em solução reduz-se de cerca de 8g/L, (eluição com NaSCN) para valores na ordem de $3 \mathrm{~g} / \mathrm{L}$ quando se utilizaram soluções de íons $\mathrm{NO}_{3}^{-}$. $\mathrm{O}$ mesmo comportamento é observado pra o cobre e para o zinco. A concentração
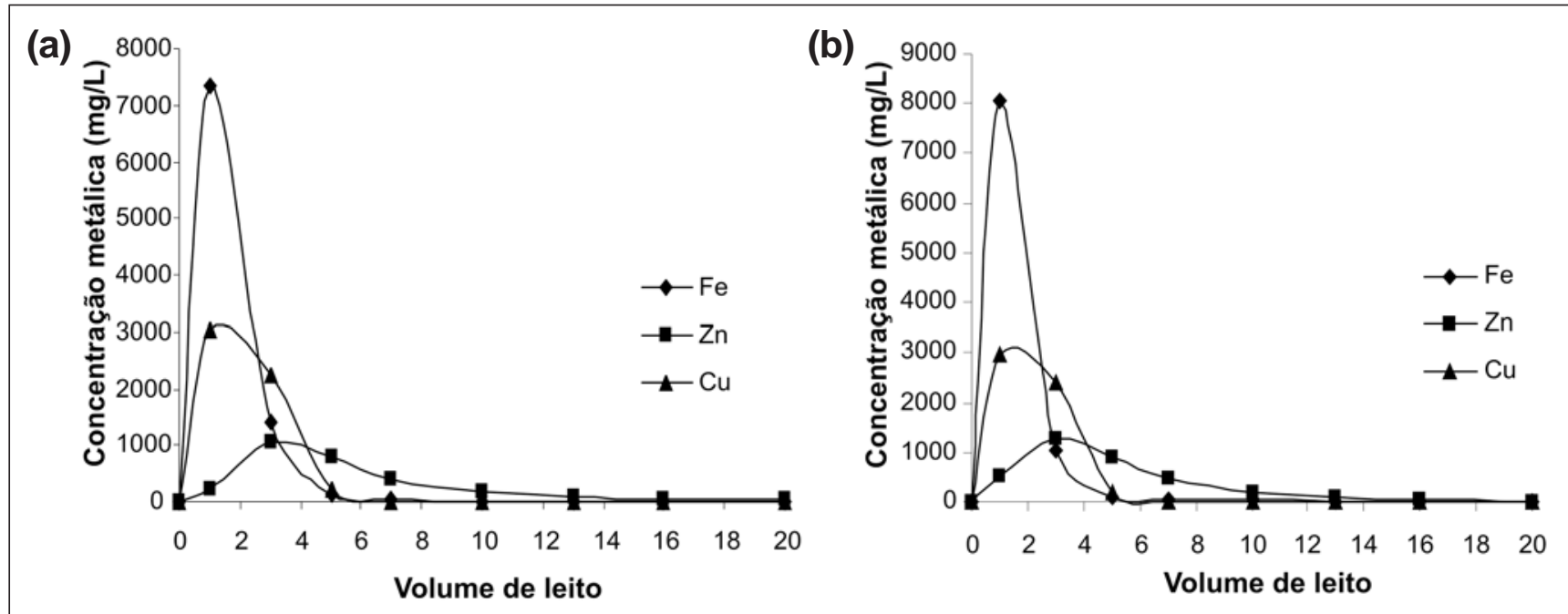

Figura 2 - Perfil de eluição da resina Bayer AP247 usando-se $1 \mathrm{~mol} / \mathrm{L} \mathrm{NaSCN}$, à temperatura de $25^{\circ} \mathrm{C}$ (a); $50^{\circ} \mathrm{C}$ (b). Concentração de cianeto livre entre 300 e 400 mg/L; pH = 11,00-11,50.

(a)

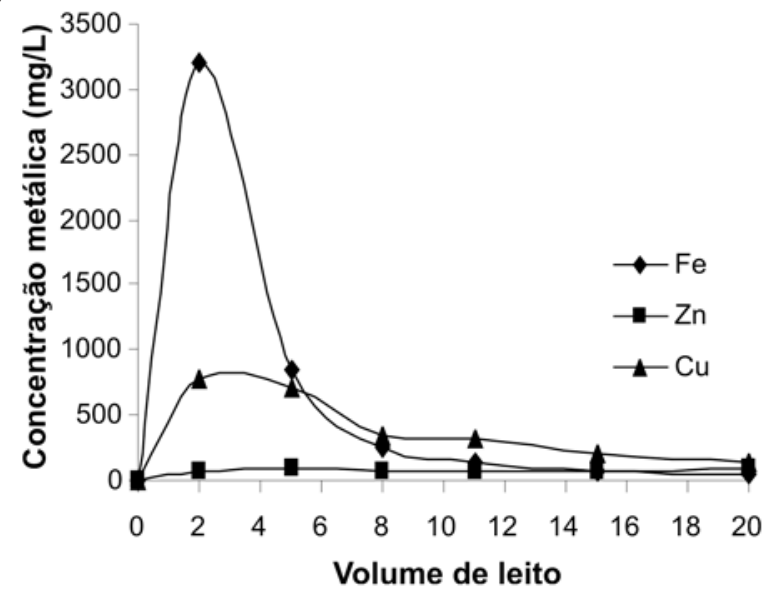

(b)

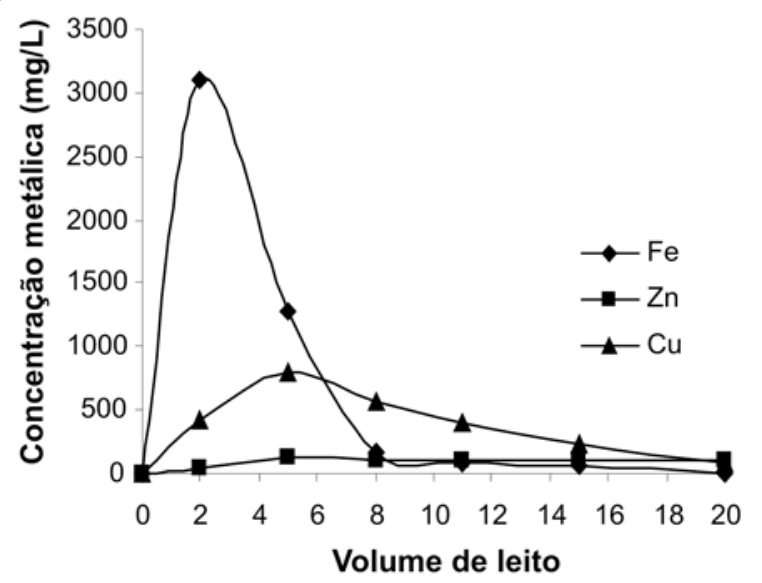

Figura 3 - Perfil de eluição da resina Bayer AP247 usando-se $1 \mathrm{~mol} / \mathrm{L} \mathrm{NaNO}$, à temperatura de $25^{\circ} \mathrm{C}(\mathrm{a})$ e $50^{\circ} \mathrm{C}$ (b). Concentração de cianeto livre entre 300 e $400 \mathrm{mg} / \mathrm{L} ; \mathrm{pH}=11,00-11,50$. 
desse último em solução é bastante reduzida (atinge o valor máximo de apenas 50mg/L). Esse comportamento reflete, ao mesmo tempo, a grande afinidade do cianeto de zinco pela resina e a baixa capacidade de eluição de soluções $1 \mathrm{~mol} / \mathrm{L}$ $\mathrm{NaNO}_{3}$. Riani et al. (2002) mostraram que mesmo em soluções 2mol/L $\mathrm{NaNO}_{3}$, a $50^{\circ} \mathrm{C}$, a eluição do zinco é, após 20 volumes de leito passados pela coluna, de $84 \%$.

Os resultados do presente trabalho mostram alguma seletividade na eluição dos metais. Por exemplo, para o tratamento de efluentes com altos teores de ferro, é possível uma eluição seletiva do metal, utilizando-se $\mathrm{NaNO}_{3}$. A $50^{\circ} \mathrm{C}$, soluções $1 \mathrm{~mol} / \mathrm{L} \mathrm{NO}_{3}{ }^{-}$eluem $90 \%$ Fe e apenas $7 \%$ Zn, após 5 volumes de leito. Por outro lado, o cobre irá acompanhar o ferro durante a eluição. Em seguida, o cianocomplexo de zinco, não removido pelas soluções de nitrato, pode ser eficientemente eluído com soluções de NaSCN.

A reciclagem de cianeto pode ser conduzida através do processo AVR, onde as letras indicam a três principais operações unitárias conduzidas no processo: Acidificação, Volatilização e Reneutralização. Basicamente, a filosofia do processo é a acidificação das soluções contendo o íon cianeto. Esse processo dissocia a maior parte dos ciancomplexos presentes no efluente. $\mathrm{O}$ cianeto livre formado é volatilizado, na forma de HCN, pela passagem de ar em contracorrente pela solução. Em seguida, essa solução é borbulhada em meio cáustico onde o HCN é convertido e convertido a íon cianeto (reneutralização). A etapa de volatilização é a mais cara, mais complexa e mais perigosa do processo (produz-se HCN gasoso) (Fleming, 2001). A possibilidade de concentração dos cianocomplexos metálicos na fase resina permite uma variante do processo AVR, na qual a etapa de volatilização do HCN é eliminada. Durante a acidificação, normalmente, tem-se a precipitação de partes dos metais (na forma de compostos como $\mathrm{Me}_{2} \mathrm{Fe}(\mathrm{CN})_{6}$, $(\mathrm{Me}=\mathrm{Cu}, \mathrm{Zn}, \mathrm{Ni})$, CuCN, CuSCN entre outros), uma vez que esses íons são comuns nos efluentes.
Nesse caso, após a acidificação da solução, realiza-se uma etapa de espessamento e filtração para remoção dos precipitados e, em seguida, a solução é neutralizada para conversão do $\mathrm{HCN}$ a $\mathrm{CN}^{-}$e recirculada na usina. Nesse caso, o processo de reciclagem de cianeto torna-se mais simples, mais seguro e de menor custo (Fleming, 2001).

\section{Conclusões}

Resinas de troca iônica base forte, são capazes de adsorver cianocomplexos metálicos de efluentes com diferentes concentrações de metais, em solução. A ordem de carregamento da resina Bayer AP247 depende da concentração em solução, sendo que esta apresentou altos carregamentos do cianocomplexo de ferro e de zinco.

NaSCN pode ser usado para eluir resinas com matriz poliacrílica, alcançando recuperações metálicas acima de 90\% para os três metais estudados. $\mathrm{O} \mathrm{NaNO}_{3}$ elui com eficiência os cianocomplexos de cobre e de ferro da resina estudada, atingindo recuperações acima de $90 \%$. Por outro lado, a eluição do cianeto de zinco é muito lenta, ou seja, atinge uma recuperação de $28 \%$ apenas depois de passados 20 volumes de leito.

Os resultados indicam que concentrações iguais a 1mol/L podem ser utilizadas na eluição do cobre e do ferro. Entretanto, nessa concentração, é recomendada a utilização de tiocianato de sódio para a eluição do zinco. O uso de resinas de troca iônica permite a concentração dos ciancomplexos metálicos, o que facilita as etapas subseqüentes do processo de reciclagem de cianeto.

\section{Agradecimentos}

Esse trabalho foi realizado com apoio da Financiadora de Estudos e Projetos - FINEP, Conselho Nacional de Pesquisa - CNPq e Governo da Bélgica. Agradecimento especial à Universidade de Liège pelo financiamento que permitiu a instalação do NVMM.

\section{Referências bibliográficas}

CIMINELLI, V. S. T. Ion Exchange Resins in the gold industry. Journal of Metals, v. 54, n. 10, p. 35, 2002.

DeVRIES, F. W. Brief overview of the baia mare dam breach. In: Cyanide: Social, Industrial and Economic Aspects. In: YOUNG, C. A., TIDWELL, L. G., ANDERSON, C. G. (eds.). New Orleans, LA, USA. Anais... TMS. 2001, p. 11-14.

FLEMING, C. A. The case for cyanide recovery from gold plant tailings positive economics plus environment stewardship. In: Cyanide: Social, Industrial and Economic Aspects. In: YOUNG, C., TIDWELL, L. G., ANDERSON, C. G. (eds.). New Orleans, LA. Anais... TMS. 2001, p. 271-288.

LEÃO, V. A., CIMINElli, V. S. T. Application of ion exchange resins in gold hydrometallurgy. A tool for cyanide recycling. Solvent Extraction Ion Exchange, v. 18, n. 3, p. 567-582, 2000.

LEÃO, V. A., CIMINELLI, V. S. T. The role of elution on the recycling of cyanide using ion exchange resins. In: Cyanide: Social, Industrial and Economic Aspects. In: YOUNG, C., TIDWELL, L. G., ANDERSON, C. G. (eds.). New Orleans, LA. Anais... TMS. 2001, pp. 303-316.

LEÃO, V. A., CIMINELLI, V. S. T., COSTA, R. S. Cyanide recycling using ion exchange resins - Application to the treatment of gold-copper ores. In: International Mineral Processing Congress, 21. MASSACI, P. (ed.). Rome, Italy. Anais... 2000, v. A, p. A6:1-9.

Leão, V. A., LUKEY, G. C., VAN DEVENTER, J. S. J., CIMINELLI, V. S. T. The effect of resin structure on the loading of copper and iron cyanocomplexes. Solvent Extraction Ion Exchange, v. 19, n. 3, p. 507-530, 2001.

LOGSDON, M. J., HAGELSTEIN, K., MUDDER, T. I. The management of cyanide in gold extraction, Internacional Concil on Metals and the Environment. 1999. 12/10, 2005.

LUKEY, G. C., VAN DEVENTER, J. S. J., SHALLCROSS, D. C. The effect of functional group structure on the elution of metal cyanide complexes from ion exchange resins. Separation Science and Technology, v. 35, n. 15, p. 2393-2413, 2000. 


\section{Tecnologia limpa para redução de impacto ambiental do cianeto na mineração de ouro}

LUKEY, G. C., VAN DEVENTER, J. S. J., SHALLCROSS, D. C. Selective recovery of gold cyanide from slurries using Highly saline water. In: VI Southern Hemisphere Meeting on Mineral Technology. In: LUZ, A. B., SOARES, P. S. M., TOREM, M. L., TRINDADE, R. B. E. (eds.). Rio de Janeiro. Anais... 2001, v. 2, p. 160-166. MILLER, G. C., PRITSOS, C. A. Unresolved problems with the use of cyanide in open pit precious metals mining. In: Cyanide: Social, Industrial and Economic Aspects. In:YOUNG, C. A., TIDWELL, L. G., ANDERSON, C. G. (eds.). New Orleans, LA, USA. Anais... TMS. 2001, p. 73-81.

RIANI, J. C., LEÃO, V. A., SILVA, C. A. Efeito da estrutura da matriz na adsorção de cianocomplexos metálicos em resinas de poliestireno. Revista da Escola de Minas, v. 57, p. 11-120, 2004.

RIANI, J. C., LEÃO, V. A., SILVA, C. A., ANJOS, A. S. Adsorção de cianocomplexos de cobre, ferro e zinco em resinas base forte. In: ENCONTRO NACIONAL DE TRATAMENTO DE MINÉRIOS E METALURGIA EXTRATIVA, 19. Anais...Recife, PE. 2002, v. 01, p. 306-311. (In portuguese with abstract in English).

RIVEROS, P. A. Selectivity aspects of the extraction of gold from cyanide solutions with ion exchange resins. Hydrometallurgy, v. 33, p. 43-58, 1993. RIVEROS, P. A., MOLMAR, R., MCNAMARA, V. M. Alternative technology to decrease the environmental impact of gold milling - a progress report on CANMET research activities in this field. CIM Bulletin, v. 86, p. 167-171, 1993.

SENGUPTA. Environmental impacts of mining, Boca Raton: Lewis Publishers. 1992. 494pp.

VACHON, D. T. Removal of iron cyanide from gold mill effluents by ion exchange. Water Science Technology, v. 17, p. 313324, 1985.

YOUNG, C. A. Cyanide: just the facts. In: Cyanide: Social, Industrial and Economic Aspects. In: YOUNG, C. A., TIDWELL, L. G. e ANDERSON, C. G. (eds.). New Orleans, LA, USA. Anais... TMS. 2001, p. 97-113.

Artigo recebido em 13/02/2006 e aprovado em 24/01/2007.

\section{Descubra as muitas informações da:}

\section{Geologia, Mineração, Metalurgia \& Materiais}

e Engenharia Civil.
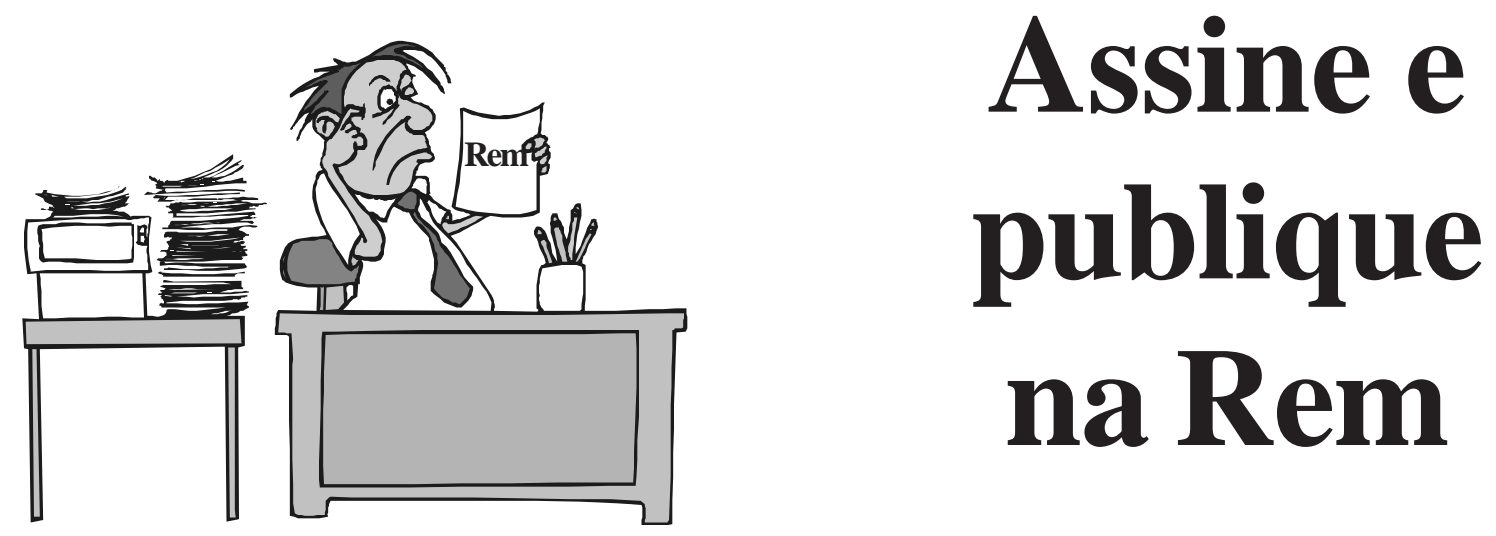

Conheça o nosso novo site: WWW.rem.com.br 